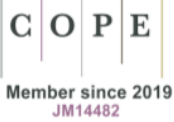

Jean-Marie Barbier*

Ewa Marynowicz-Hetka**

\title{
Entrer dans la « pensée transformation »
}

\begin{abstract}
Ce texte présente le contenu du numéro thématique consacré à une réflexion sur « la pensée transformation ", en lien avec les processus de professionnalisation, et de plus généralement avec les transformations simultanées des activités et des sujets par et dans les activités.
\end{abstract}

Mots-clés: transformation, pensée transformation, professionnalisation, travail collectif.

\section{“Thinking Transformations" - an Introduction to the Issues}

\begin{abstract}
The text presents the content of the thematic issue devoted to the reflection on the issue of "thinking transformations", related to the processes of professionalization and, more generally, to the interrelated transformations of activities and entities occurring in and through their activities.
\end{abstract}

Keywords: transformations, thinking transformations, professionalization, teamwork.

«Panta rei », tout s'écoule. C'est à Héraclite, à l'aube d'une culture occidentale pourtant caractérisée par les démarches d'appréhension des objets du monde en termes d'objets de pensée provisoirement stabilisés (Jullien 2009 : 17-21; Jullien 2017 : 181-185), qu'est attribuée la première caractérisation de la labilité, de la

* Conservatoire National des Arts et Métiers, Chaire Unesco - ICP, Paris, France.

** Université de Lodz. Pologne.

Article soumis le 18 juin 2021 ; accepté pour publication le 22 juin 2021. 
transformation constante des environnements dans lesquels les êtres humains vivent et agissent.

Cette même intuition accompagne l'histoire même des sciences modernes puisqu'un auteur aussi important dans la constitution d'une discipline que Lavoisier en chimie admet également que : «Rien ne se crée, ni dans les opérations de l'art, ni dans celles de la nature, et l'on peut poser en principe que, dans toute opération, il y a une égale quantité de matière avant et après l'opération ; que la qualité et la quantité des principes est la même, et qu'il n'y a que des changements, des modifications » (Lavoisier 1789).

Les coordonnateurs et auteurs de ce numéro font l'hypothèse que le mouvement contemporain de professionnalisation affectant l'enseignement supérieur peut réactiver toutes les questions liées au statut des transformations dans le rapport que l'homme entretient avec le monde, et avec lui-même vivant et agissant :

- Les différents environnements de l'être humain se transforment de façon continue : même les "sciences de la terre » font l'hypothèse de transformations certes plus lentes, mais fondamentales pour approcher le monde objet d'action ;

- Un grand nombre des activités humaines s'analysent précisément comme des activités de transformation du monde, se distinguant par les objets sur lesquels elles prétendent agir, ce qui fonde tout à la fois les disciplines professionnelles (les techniques ou disciplines de transformation du monde) et les disciplines académiques se donnant précisément comme objet la connaissance des objets de transformation (sciences dites « exactes »);

- Avec la tertiarisation des activités et le développement des 'métiers de l'humain', un grand nombre de disciplines se donnent même comme objet la transformation des sujets en activité: sciences de l'éducation, sciences de la communication, sciences du management, intervention sociale etc. ;

- Enfin en agissant et en transformant le monde, les sujets humains se transforment eux-mêmes et ces transformations sont le plus souvent silencieuses.

Produit d'une collaboration de plusieurs années entre deux équipes polonaise (Université de Lodz en association avec Académie de Poméranie à Słupsk et l'Université de Białystok), et française (Cnam-Formation et apprentissages professionnels et chaire Unesco Formation Professionnelle, Construction personnelle et Transfomations sociales), et bénéficiant du croisement des deux cultures, ce numéro thèmatique a précisément pour objectif d'explorer les différents aspects épistémologiques, théoriques, méthodologiques, sociaux de ce qui pourrait être appelé «la pensée transformation » en particulier lorsqu'elle aborde la question des actions professionnelles et de la formation professionnelle.

Il pose d'épineuses questions, à rebours des habitudes d'action et des habitudes intellectuelles :

- comment fonctionne une approche dynamique, reconnaissant la prééminence du 'flux' de la vie ; 
- de quels outils se dote une approche contextuelle, reconnaissant que le sens de l'action se constitue de façon locale et temporaire?

- y a-t-il un primat de la pratique sur la théorie ?

- comment penser les transformations simultanées des organisations, des sujets, des perceptions, des représentations, des jugements, des raisonnements ?

- comment appréhender l'activité en train de se faire ?

Pour y répondre, nous proposons 15 articles structurés dans trois parties: Études et discours ; Travaux de recherche ; Discussion/polémiques/critiques. Dans la première partie se trouvent les articles qui analysent les différents aspects du paradigme de la transformation : Jean-Marie Barbier parle des liens conjoints entre action professionnelle et pensée transformation; Ewa Marynowicz-Hetka présente le concept d'analyse transversale des activités de Jean-Marie Barbier (2017), un aperçu de point de vue de pédagogie sociale et propose le paradigme de transformation ; Lech Witkowski, dans une large perspective philosophique, présente le concept de transformations, soulignant ses dominantes et la spécificité de l'espace entre la dynamique et la structure de la processualité ; Anna Walczak, en se référant aux multiples philosophes français analyse la question d'identité d'un sujet agissant, en soulignant la catégorie de différence.

Dans la deuxième partie du numéro, nous avons placé les articles produits sur la base de matériaux de recherches qualitatives, précisément narratives, analyse de discours, observation ethnographique. Ils portent sur l'analyse des dynamiques des activités et des transformations en cours d'action. Ces articles embrassent les deux phénomènes situés : les transformations et les interventions, et souvent se réfèrent aux concepts d'activité et de transformation de Jean-Marie Barbier et d'autres chercheurs de même épistémologie. L'espace d'analyse concerne plutôt la sphère professionnelle des personnes qui exercent les métiers humains/sociétaux dans les secteurs de la santé (Martine Dutoit), de l'action syndicale (Marie Jasmine Hyppolite), de la police (Myriam Léonard), du troisième secteur (économie sociale, Daniela Rodriguez), des entreprises (Kim Vu), de l'aide familiale (Izabela Kamińska-Jatczak), et aussi de la sphère privée, de la famille (Katarzyna Gajek).

Dans la troisième partie du numéro, nous proposons les articles qui entreprennent des discussions ou des polémiques avec les concepts mentionnés dans ce numéro. Ces articles sont préparés sur la base des rencontres et sur l'analyse de discours décrivant les circonstances des flux intellectuels entre nos deux cultures de pensée : française et polonaise (Marcin Kafar, Ewa Marynowicz-Hetka) ; qui donnent un reflet sur l'analyse transversale des activités (Barbier 2016) en tant qu'expérience signficative de recherche (Izabela Kamińska-Jatczak), et montrent une possibilité d'utiliser des outils d'analyse des activités (Barbier 2016) lorsque la créativité et les émotions deviennent objet de pensée, et font des espaces de transformation et de synergie (Halina Monika Wróblewska). L'article de Lech Witkowski, effet de lecture d'un ouvrage collectif Représenter/transformer (Barbier, Durand 2018), souligne de façon transversale le rapport représentations/transformations. 
Dans la quatrième partie du numéro intitulé : "Les actualités », nous proposons aux lecteurs deux textes: le premier préparé par Jean-Marie Barbier et Martine Dutoit sur les activités actuelles de la chaire Unesco ICP «Formation Professionnelle, Construction Personnelle, Transformations sociales», en tant qu'exemple du programme coordonné de recherche Se préparer à l'action, ayant pour finalité de mettre en relation des chercheurs et des professionnels intéressés à la construire de «possibles activités »; le second, préparé par Izabela Kamińska-Jatczak et Ewa Marynowicz-Hetka, passe en revue des principales lignes de la longue coopération entre Jean-Marie Barbier et l'Université de Lodz, Chaire de pédagogie sociale. Le texte montre le processus de partage de l'approche épistémologique, et plusieurs activités autour de la catégorie : "pensée transformation », qui ont préparé les auteurs de ce numéro à l'idée de ce sujet, exprimée en formule commune et partagée, sans oublier l'expression de leurs différences.

A l'image de son objet, ce numéro a permis, par la confrontation des points de vue une transformation des représentations des auteurs eux-mêmes.

Porté par Nowis, ce numéro a adopté aussi des méthodes de travail susceptibles d'assurer transversalité culturelle et communication scientifique internationale.

Bonne lecture!

\section{Bibliographie}

Barbier J.-M. (2016) Leksykon analizy aktywności. Konceptualizacje zwyczajowych pojęć, trad. E. Marynowicz-Hetka, Łódź, Wydawnictwo Uniwersytetu Łódzkiego.

Barbier J.-M. (2017) Vocabulaire d'analyse des activités. Penser les conceptualisations ordinaires, Paris, Presses Universitaires de France.

Barbier J.-M., Durand M. (eds.) (2018) Représenter/transformer, Débats en analyse des activités, coll. C. Cohen, M.-L. Vitali, Paris, L’Harmattan.

Jullien F. (2009) Les transformations silencieuses, Paris, Grasset.

Jullien F. (2017) Une seconde vie. Commencer véritablement d'exister, Paris, Grasset.

Lavoisier A. (1789) Traité élémentaire de chimie, Paris, Cuchet.

\section{Pour citer cet article}

Barbier J.-M., Marynowicz-Hetka E. (2021) Entrer dans la " pensée transformation », „Nauki o Wychowaniu. Studia Interdyscyplinarne” 2(13), 11-14, https://doi.org/ 10.18778/2450-4491.13.01 